\title{
The Study on Detection Method of Water Vapor on Boundary Layer Based on Multiagent System
}

\author{
Xu Dongdong, Zhou Jie, Xia Jingming, Lu Zhenyu, and Huan Hai \\ Nanjing University of Information Science \& Technology, Jiangsu 210044, China \\ Correspondence should be addressed to Zhou Jie; zhoujie45@hotmail.com
}

Received 12 July 2014; Revised 25 September 2014; Accepted 2 October 2014

Academic Editor: Wei Zhang

Copyright (C) $2015 \mathrm{Xu}$ Dongdong et al. This is an open access article distributed under the Creative Commons Attribution License, which permits unrestricted use, distribution, and reproduction in any medium, provided the original work is properly cited.

\begin{abstract}
A method of detecting water vapor on boundary layer based on multiagent system is proposed in this paper. Multiagent system receives electromagnetic signals emitted by the telecommunication base station. Due to the analysis of the actual electromagnetic wave signal propagation path in the atmosphere, atmospheric refraction index and moisture inversion are discussed in this paper. And the feasibility of using electromagnetic detection method is also analyzed. A multiagent system is designed to receive the electromagnetic signals. The composition and function of the multiagent system are clearly described. The atmospheric refractivity is detected by the multiagent system in three weather conditions of sunny, foggy, and rainy days. The results demonstrate the feasibility of water vapor detection method of multiagent system boundary by comparing the result of experiment with traditional method.
\end{abstract}

\section{Introduction}

Water vapor is a kind of trace gas and mainly distributes in the bottom of the atmosphere. In particular when the height is less than 10-12 kilometers, water vapor accounts for $99 \%$ of all total. Even though the content is very small, it is the most active part in the atmosphere and is of great significance to life on Earth. Water vapor presents three states in the atmosphere varied from the temperature and is the material basis for the formation of clouds and rainfall. Water on Earth circulates between the atmosphere, land, and ocean and atmospheric water vapor plays an important role in the global water cycle. Most phenomena are the results of the changes of atmospheric water vapor while in the phase transition process water vapor absorbs and releases large amounts of latent heat which directly have impact on temperature of the ground and the air, hence affecting the stability of the atmosphere and the formation and evolution of atmospheric vertical stability and convective weather systems. Water vapor is a changeable parameter and plays a very important role in the atmospheric energy transfer and the evolution of weather systems. Atmospheric water vapor content is an essential parameter for forecasting rainfall of mesoscale or local scale [1]. Hence, obtaining atmospheric water vapor information in high precision and high space-time resolution is very important for accurately analyzing the formation and evolution of mesoscale severe weather system and mediumscale severe weather forecasting and disaster mitigation.

Factors in atmosphere which affect the distribution and water vapor content are very complex, and meanwhile the space-time change of water vapor is the most obvious factor in the atmosphere. Currently, the detection and requirement of water vapor data of high space-time resolution are still a difficult problem due to the limitation of detection funds, networking, and techniques. However, the water vapor data of high space-time resolution is the prerequisite for accurate numerical prediction. Hence, applying new technologies to improve the accuracy and resolution of water vapor observation is the most active research interest in the area of atmospheric sounding. Currently, the effective methods which have been used in sensing of atmospheric water vapor are the observation of hygrometers of ground weather station, solar spectrum water vapor analyzer, meteorological aircraft detection, laser detection, radar detection, satellite observation of water vapor, ground-based microwave radiometers detection, radio water vapor detection technology, and GPS measurements of water vapor. Among these methods, radio water vapor detection technology is the main mean which 
is used in the high altitude meteorological data detection at present. The shortages of this method are that the cost of radiosondes is high and they have lower network distribution density. Meanwhile, the temporal resolution of observation data is very low which is not enough to distinguish the rapid space-time variation of water vapor. Furthermore, because of the restriction of sounding balloon tracking and locating technology as sounding balloon has low location resolution under high altitude complex turbulent condition and during the period of thunderstorms, we cannot detect the variation of water vapor during the process of convective weather effectively. The GPS used for measuring water vapor is a new method in atmospheric detection. The disadvantage of GPS measurement system is that the cost is high and it is hard to achieve high density distribution network. High resolution numerical weather prediction model of next generation needs meteorological date of 2-5 kilometers when forecasting mesoscale severe weather. However, GPS detection cannot satisfy this requirement $[2,3]$.

This paper provides a method which applies multiagent into the area of weather forecast and detection of water vapor content of atmospheric boundary layer. Multiagent receives electromagnetic signals from the communication base station. Then the atmospheric refractivity can be derived by using signal refraction phenomenon which resulted from the transmission of radio signals in the atmosphere. Since the atmospheric refractivity is a function of temperature, pressure, and water vapor pressure, the water vapor content can be derived from atmospheric refractivity under the assumption that the pressure and temperature are known [4]. Multiagent receiving signal from communication base station is a measurement method of a passive way. When the agent receives electromagnetic signal, multiple agents communicate with each other and sample the current location. Through the decision-making mechanism, the agent receives electromagnetic signal again after altering the location [5]. This method has its own superiorities compared with traditional detection methods.

(1) The method can be based on exiting communication network, such as mobile communication network and digital broadcasting network. Hence the observation network does not need to be reconfigured, and the cost of the observation could be decreased.

(2) Electromagnetic signal transmits linearly or quasilinearly on the boundary layer without ionosphere reflection. Hence, there is no ionosphere delay interference phenomenon and the observation accuracy can be higher.

(3) Different transmission characteristics of electromagnetic signals of different wavelength can be used for enriching observation data.

(4) The time interval of multiagent receiving electromagnetic signals can be altered, which extends temporal resolution.

(5) Multiagent communication between the data generated according to the location information combined data.
TABLE 1: The level of sensitivity of electromagnetic waves of different wavelength to the water vapor.

\begin{tabular}{lcc}
\hline $\begin{array}{l}\text { Frequency } \\
(\mathrm{MHz})\end{array}$ & $\begin{array}{c}\text { Wavelength } \\
(\mathrm{m})\end{array}$ & $\begin{array}{c}\text { The level of sensitivity } \\
\left(\mathrm{km}^{-1} \mathrm{~g}^{-1} \mathrm{~m}^{3}\right)\end{array}$ \\
\hline 222 & 1.351 & 1.6 \\
900 & 0.333 & 6.5 \\
1800 & 0.166 & 13.01 \\
\hline
\end{tabular}

(6) Data handling ability of multiagent individual can preprocess data, making it easy to post data processing.

\section{Feasibility Analyses for Detection Method of Water Vapor of Boundary Layer Based on Multiagent}

When propagating in the atmospheric environment, electromagnetic wave of different frequency has different level of sensitivity to water vapor which depends on the wavelength. Table 1 shows the level of sensitivity of electromagnetic waves of different wavelength to the water vapor [6].

From Table 1, it is obvious that the shorter the wavelength of electromagnetic wave, the higher the sensitivity of electromagnetic waves to the water vapor.

Electromagnetic wave is transported linearly in vacuum at a speed of $3 \times 10^{8} \mathrm{~m} / \mathrm{s}$. The electromagnetic wave will propagate in curve obviously which is called atmospheric refraction, when transmitted in the atmosphere, especially in remote transmission and atmospheric meteorological elements have abnormal vertical distribution. The index of atmospheric refraction has a very close relation to dielectric constant of atmosphere which depends on the material density, temperature, and the properties of molecules. The temperature, humidity, and atmospheric pressure vary along with molecules.

Electromagnetic wave has different velocity in inhomogeneous medium which is due to different physical properties of the propagation medium forming different levels. The essence of refraction is the deflection of electromagnetic wave in the propagation direction when the direction is not perpendicular to interface of medium. Investigating plane wave using Maxwell equation set can derive that

$$
v=\frac{1}{\sqrt{\varepsilon \mu}},
$$

where $v$ is the propagation velocity of electromagnetic wave in some homogeneous medium. $\varepsilon$ and $\mu$ are absolute dielectric constant and absolute permeability, respectively. In a vacuum,

$$
v=c=\frac{1}{\sqrt{\varepsilon_{0} \mu_{0}}}=2.9979 \times 10^{8} \mathrm{~m} / \mathrm{s},
$$

where $\varepsilon_{0}=8.85 \times 10^{-12} \mathrm{C} \mathrm{kg}^{-1} \mathrm{~m}^{-1}$ and $\mu_{0}=1.2566 \times$ $10^{-6} \mathrm{C} \mathrm{kg}^{-1} \mathrm{~m}^{-1}$. 
The refractive index of medium $n$ usually can be defined as

$$
n=\frac{c}{v}=\sqrt{\frac{\varepsilon_{0} \mu_{0}}{\varepsilon \mu}}=\sqrt{\varepsilon_{r} \mu_{r}} .
$$

The magnetic permeability of most media satisfies $\mu_{r} \approx 1$; hence $n^{2}=\varepsilon$.

It should be noted that the most common form of refractive index is a complex number as

$$
m=n-k i,
$$

where $m$ is usually called complex refractive index. The real part $n$ of $m$ is ordinary refractive index and the imaginary part $k$ of $m$ is a coefficient which denotes the attenuation of electromagnetic wave signal. In this paper, we do not consider attenuation and use the refractive index $n$.

Considering the polarization of atmosphere in external electric field, when the medium consisting of nonpolar molecules is polarized, its molecules form an electric dipole of electric dipole moment $\vec{p}$. And $\vec{p}$ is proportional to the electric field which acts on molecules

$$
\vec{p}=\beta \vec{E}^{\prime} \approx \beta \vec{E}
$$

where $\beta$ is the polarizability of molecules. $\vec{E}^{\prime}$ denotes effective electric field acting on molecules. $\vec{E}$ is the average electric field. It can be approved that the relationship between $\beta, \vec{E}^{\prime}$, and $\vec{E}$ can be written as

$$
\begin{gathered}
\vec{E}^{\prime}=\vec{E}+\frac{4}{3} \pi \vec{p}=\frac{1}{1-(4 / 3) \pi \beta}, \\
\vec{p}=\frac{\beta \vec{E}}{1-(4 / 3) \pi \beta} .
\end{gathered}
$$

For polar molecules such as water vapor molecules, the electric dipole moment $\vec{p}$ can be derived into two parts as $\vec{p}=\overrightarrow{p_{0}}+\overrightarrow{p_{1}}$ where $\overrightarrow{p_{0}}$ is related to the orientation of polar molecules and has no relation to external field. Hence, $\overrightarrow{p_{1}}$ can be written as

$$
\overrightarrow{p_{1}}=\frac{\beta \vec{E}}{1-(4 / 3) \pi \beta} .
$$

If the number of molecules per unit volume is $N$, then the total dipole moment $\vec{p}$ can be written as

$$
\vec{p}=N \vec{p}=N \beta \vec{E}^{\prime}=\frac{N P \vec{E}}{1-(4 / 3) \pi N \beta} .
$$

The intensity of electric induction in isotropic medium is

$$
D=\varepsilon \vec{E}=\vec{E}+4 \pi \vec{p}
$$

Then we can derive the relationship between dielectric constant and molecular polarizability as

$$
\frac{\varepsilon-1}{\varepsilon+2}=\frac{4}{3} \pi N \beta
$$

In the air comprising of multiple polar molecules, when external electric field $\vec{E}=0$, the sum of inherent dipole moment equals 0 for random orientations. When external electric field $\vec{E} \neq 0$, natural pole moment of each molecules makes contribution to total dipole moment with certain probability. And the probability relates to absolute temperature $T$ in the atmosphere. To quantify the relationship between the polarization of medium of polar molecules and the temperature, according to the statistical theory of Boltzmann, the number of polar molecules between $U$ and $U+d u$ in the energy per unit volume is as follows:

$$
d N=C e^{U / k T} d U,
$$

where $k$ is the Boltzmann constant, $T$ is the absolute temperature of the atmosphere, and $C$ is a proportional constant. It is known that the electrostatic energy of electric dipole with electric dipole moment $\vec{p}$ is

$$
U=-p E \cos \theta
$$

where $\theta$ is the angle between electric axis and electric field $\vec{E}$. $p$ and $E$ are modulus of $\vec{p}$ and $\vec{E}$. Considering the polarization intensity of medium of polar molecules, we should take rotary and slight elastic deformation of each molecular dipole in external field into consideration. Hence, under the condition of weak electronic field and normal temperature, $\vec{p}$ can be expressed as

$$
\frac{\varepsilon-1}{\varepsilon+2}=\frac{4 \pi N}{3}\left(\beta+\frac{p^{2}}{3 k T}\right) .
$$

The atmosphere consists of nonpolar molecules of dry air and polar molecules of water vapor. Hence, under the action of electric field, the electric polarization vector $\vec{p}$ of mixed gas can be expressed as

$$
\vec{p}=\overrightarrow{p_{n}}+\overrightarrow{p_{p}}=K_{n} \vec{E}+K_{p} \vec{E}
$$

where $\overrightarrow{p_{n}}, \overrightarrow{p_{p}}, K_{n}$, and $K_{p}$ denote polarization vectors and polarizabilities of nonpolar and polar molecules. Without regard to the difference between $\vec{E}$ and $\vec{E}^{\prime}$, the polarizability $K$ of mixed gas can be written as

$$
K=N_{n} \beta_{n}+N_{p}\left(\beta+\frac{p^{2}}{3 k T}\right) .
$$

It is known that the relationship between polarizability $K$ and dielectric constant $\varepsilon$ is

$$
\varepsilon-1=4 \pi K
$$

While $\varepsilon^{2}-1=n^{2}-1 \approx 2 n-1$, we can find that

$$
n-1 \approx 2 \pi\left(N \beta_{n p}+N_{p} \frac{p^{2}}{3 k T}\right),
$$

where $N=N_{n}+N_{p}$ denotes the number of molecules of mixed gas per unit volume and $\overrightarrow{\beta_{n p}}\left(\left(N_{n} \beta_{n}+N_{p} \beta_{p}\right) /\left(N_{n}+N_{p}\right)\right)$ 
is the average number of polarizations of molecules of mixed gas.

The atmosphere is a mixture of gases and the atmospheric pressure is $P=P_{d}+e$. Assuming that the density is $\rho$, gram molecular weight is $\mu$ and ideal gas state equation per unit volume is $P=(\rho / \mu) R T$; according to the equation $\rho=N \mu$, it can be derived that

$$
N=\frac{P}{R T}
$$

where $P$ is the atmospheric pressure and its unit is hPa. $R$ is universal gas constant with the value of $8.31 \mathrm{~J} \cdot \mathrm{mol}^{-1} \cdot \mathrm{k}^{-1}$.

Similarly, the number of molecules of water vapor per unit volume $N_{p}$ can be derived as

$$
N_{p}=\frac{e}{R T}
$$

where $e$ is the water vapor pressure and its unit is $\mathrm{hPa}$. Furthermore, it can be derived as

$$
(n-1) 10^{6}=\frac{A}{T}\left(P+\frac{B e}{T}\right),
$$

where $A$ and $B$ are coefficients which are determined by experiments, $T$ is the absolute temperature, and the units of $P$ and $e$ are hPa.

While the value of $n$ is small and bad for expression, the refraction unit $N$ is always used actually which can be called refraction index $N$ :

$$
N=(n-1) 10^{6}
$$

The refraction index $N$ includes dry refractive components caused by atmospheric pressure $P$ and temperature $T$ and wet refractive components caused by water vapor pressure. The expression of atmospheric refractive index can be derived after decomposing dry and wet refractive components as

$$
N=77.6 \frac{P}{T}+3.75 \times 10^{5} \frac{e}{T^{2}}=N_{\text {dry }}+N_{\text {wet }},
$$

where the first item $N_{\text {dry }}$ is just connected with atmospheric pressure $P$ and temperature $T$ and the second item $N_{\text {wet }}$ which is called wet refractive component is related to water vapor pressure. The variation range of $N_{\text {dry }}$ is between 225 and 325 units and $N_{\text {wet }}$ is between 0 and 150 units. And the change of humility has larger impact on refractive index than atmospheric pressure and temperature. The investigation in this paper is an inverse process actually which takes advantage of transmission feature of electromagnetic wave in the atmosphere to inverse refractive index. Then the value of water vapor pressure or the distribution of water vapor can be derived due to refractive index, temperature $T$, and atmospheric pressure $P$ [7-9].

However the value of $N$ during the actual inversion of water vapor cannot be obtained directly and it needs the comparison between the actual broadcasting distance and visual broadcasting distance (pseudorange) between electromagnetic transfer and receiver position. Lower atmosphere effects

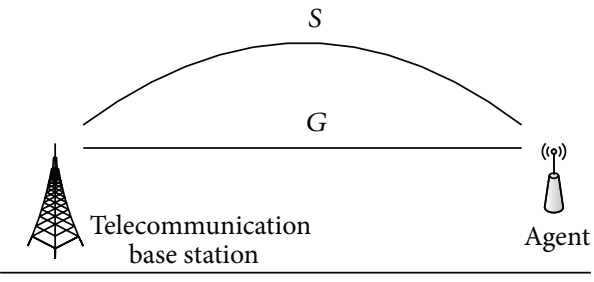

FIgURe 1: Propagation path $S$ of electromagnetic wave at the bottom of the atmosphere and distance $G$ between agent and base station.

the transmission of electromagnetic wave in two ways, while the signal is transported near the ground and on the boundary layer. First, comparing to vacuum, the broadcasting velocity of electromagnetic wave becomes slower. The relationship between the velocities of electromagnetic wave in atmosphere and in vacuum is $n=c / v$, where $n$ is the atmospheric refractive index. Second, the propagation path changes from linear to curved. These two influences are caused by the change of atmospheric refractive index $N$ along with the propagation path. The delay of propagation of signal compared with vacuum equals the increase of propagation path length [10]. Therefore, the distance between agent and base station which is calculated according to the propagation delay and velocity is not the real distance actually but the visual broadcasting distance which is called pseudorange. Figure 1 shows the propagation path $S$ of electromagnetic wave at the bottom of the atmosphere and distance $G$ between agent and base station.

The increased propagation path can be expressed as

$$
\Delta L=c \Delta t-G=c \int_{L} \frac{d s}{v}-G=\int n(s) d s-G,
$$

where $c$ is the speed of light in vacuum, $\Delta t$ is the actual propagation time of signal, $v$ is the propagation velocity of signal in the atmosphere, and $n(s)$ is the average refractive index of the signal on the transmission path. The integral is performed along with the curved path of propagation of electromagnetic signals. $G$ is the actual geometric distance between the transfer and the receiver stations. The formula can be rewritten in the following form:

$$
\Delta L=\int_{L}[n(s)-1+1] d s-G=\int_{L}[n(s)-1] d s-(S-G),
$$

where $S$ is the length of propagation path. The first item is a slower one and is only related to atmospheric index $n$ which is inversely proportional to the propagation velocity $v$. The second item is a signal bending one which is the difference between signal bending path $S$ and linear propagation path $G$. The increasing of propagation path is mainly caused by signal slowing effect. Replacing atmospheric refractive index with refractivity, we can find that

$$
\Delta L=10^{-6} \times \int_{L} N(s) d s .
$$




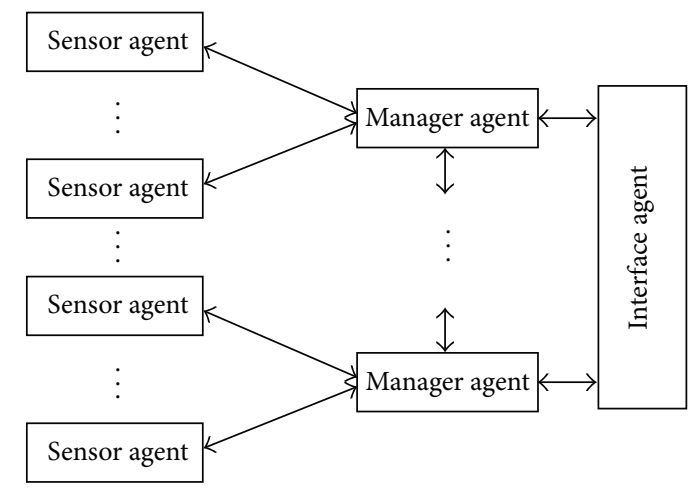

FIGURE 2: The architecture of multiagent system.

And $\Delta L$ can be decomposed as follows:

$$
\Delta L=10^{-6} \times \int_{L}\left(N_{\mathrm{dry}}+N_{\text {wet }}\right) d s=\Delta L_{d}+\Delta L_{w},
$$

where $\Delta L_{d}$ and $\Delta L_{w}$ denote dry delay and wet delay, respectively. Compared with GPS system which is used in water vapor measurement, multiagent system could measure atmospheric refractive index of lower or boundary layer. Without ionospheric propagation of signal, it is not necessary to use the double-frequency method to correct the error caused by ionospheric delay which reduces the possibility of measurement error to some extent. In addition, multiagent detection system measures water vapor in low atmosphere and the water vapor content per volume is bigger than using GPS measurements which measure the whole atmospheric convection layer. Hence, it will cause larger delay under the same propagation distance. Besides, according to the measured refractive index $N$ which is used to inverse water vapor pressure or intensity near the ground or boundary layer, we only need to combine it with observation data of atmospheric pressure and temperature near the ground and gradient distribution near the ground or boundary layer [11]. Hence, there is no need to consider the weighted average temperature of the whole atmosphere which makes a further error reduction of the source.

Therefore, the key of the inversion of atmospheric refraction index is to find the propagation path delay $\Delta L$. The key issues of finding $\Delta L$ are to obtain signal propagation delay $\Delta t$ in high accuracy and to eliminate error caused by noise and so forth [12].

\section{The Architecture of Multiagent System}

A multiagent system is designed to detect the vapor on boundary layer by calculating the refractive index of electromagnetic waves. The system has three components, such as sensor agent, manager agent, and interface agent. Figure 2 shows the architecture of multiagent system.

3.1. Sensor Agent. Sensor agent is the basic component of the multiagent system. The main task of sensor agent contains the following:
(1) receiving the electromagnetic waves of current position,

(2) acquiring the relevant information and data of current position,

(3) getting the information and data of current environment,

(4) calculating the corresponding water vapor content,

(5) uploading all data to manager agent.

There are some rules to help sensor agent work effectively:

(i) time resolution rules, which are used to control the time resolution of receiving the electromagnetic wave signal according to the preestablished time resolution or changed by instructions,

(ii) data acquiring rules, which are used to acquire the parameters of current position and environment such as GPS data, temperature, relative humidity, wind direction, wind speed, rainfall, air pressure, and solar radiation,

(iii) vapor calculating rules, which are used to set the different algorithms of calculating,

(iv) sensors connecting rules, which are used to connect with manager agent and other sensors agents nearby for data communication.

Sensor agent could only receive the very electromagnetic waves from telecommunication base station generally. But it also could receive several electromagnetic waves from different telecommunication base stations in other cases. When it happens, the sensor agent would provide several data. Each sensor agent associates with other sensor agents.

3.2. Manager Agent. All data collected from sensor agents upload to manager agent. The main task of manager agent contains the following:

(1) verifying the data of water vapor according to position information,

(2) sampling the verified data,

(3) merging the data based on position information,

(4) uploading all preprocessed data to interface agent,

(5) altering the frequency of sending data through the control instruction from the interface agent.

Some rules are used to make manager agents cooperate with each other:

(i) correlation rules, which are used to detect correspondences between data provided by several sensors to evaluate the quality of these data,

(ii) data sample rules, which are going to be used to set the different algorithms of data sampling,

(iii) data merge rules, which are used to merge the data from sensor agents with the data acquired in traditional method or historical data, 
(iv) communication rules, which are used to define the communication protocol among sensor agents and interface agent.

The manager agent would standardize the data received from sensor agents and exclude the wrong data by comparing the data based on same position or nearby. Then the manager agent would get the valid data. Atmospheric water vapor content generally existed in the form of a continuous function, so the character of data acquired in same position or nearby should also show continuity [13]. This requires the use of the data associated with the agent.

The valid data acquired by sensor agents represent the character of selected area. But it is not necessary to analyse all valid data. So some data would be sampled from the valid data according to position information, which could also represent the character of selected area and distribute evenly. Here we use the method of stratified sampling. Firstly we stratify the sample space, then random sample at each layer [14]. Location-based sampling method is implemented as following.

(I) Stratification. The sample space is divided into nonoverlapping subspaces, called the layer of each subspace. In telecommunication base station as the center, the coverage is divided into several rings with the radius of $r$. Set distances $d_{1}, d_{2}, d_{3}, \ldots, d_{k}$ with the number of $k$ to determine the space of each layer [15].

(II) Random Sampling. Randomly sample at each layer. Determine the number of each layer $n_{1}, n_{2}, n_{3}, \ldots, n_{k}$ and make sure the result of sample distributes evenly. Figure 3 shows the position of sensor agents and telecommunication base station and the sampling based on position.

The sample number in layer $i$ is as follows:

$$
n_{i}=n_{e} \times \frac{s\left(d_{i}\right)}{\sum_{j=1}^{k} s\left(d_{j}\right)} .
$$

And $n_{e}$ is the presetted sample number and $s\left(d_{i}\right)$ is the mumber of sample covered in layer $i$ :

$$
\begin{aligned}
& S\left(d_{1}\right)=R Q\left(r_{1}\right), \\
& S\left(d_{2}\right)=R Q\left(r_{2}\right)-R Q\left(r_{1}\right), \ldots, \\
& S\left(d_{i}\right)=R Q\left(r_{i}\right)-R Q\left(r_{i-1}\right), \ldots, \\
& r_{1}=d_{1}, \quad r_{2}=r_{1}+d_{2}, \ldots, \quad r_{i}=r_{i-1}+d_{i}, \ldots
\end{aligned}
$$

The parameters $R Q\left(r_{i}\right)$ could be inquired by the range of sensor agents. Euclidean distance query and network distance query depend on the distribution of sensor agents. The range query refers to a query $q$ where $Q$ is the center point, a radius equal to the length of a subset of the query objects within a circle of radius $r$. In order to improve the efficiency of range query, algorithm of LUR-tree is used to build the index for the moving stored objects $[16,17]$.

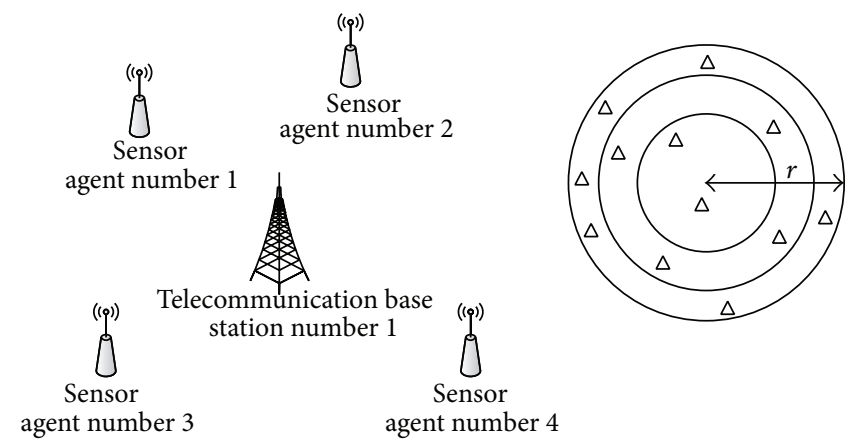

FIgURE 3: The position of sensor agents and telecommunication base station sampling based on position.

3.3. Interface Agent. The interface agent is the humancomputer interface between human and multiagent. The main task of interface agent contains the following:

(1) showing the current position and status of all sensor agents on the map,

(2) setting the parameters of the system.

All valid data which are preprocessed by agents would upload to interface agent. The system's interface uses web application that displays all valid data and the status of all agents via Maps in Time [18]. The frequency of update valid date would be set by different time resolution.

\section{Implementation}

The system has been implemented using a JADE platform [19]. JADE is a software framework to develop agent applications in compliance with FIPA specifications for interoperable intelligent multiagent systems. The development of multiagent system greatly simplifies by middleware. The inference engine for the rule-based system has been implemented using Drools [20].

In experiment, the sensor agents are settled on several vehicles, and the manager agents are placed in fixed position among the selected observation area. The vehicles select roads randomly during the processing of observation. The time resolution of uploading data is controlled by the instruction of interface agent.

Three different weather conditions are selected to verify the results of the measurement accuracy with the method of multiagents under sunny, foggy, and rainy weather. The atmospheric refraction index is calculated by the measured electromagnetic signals. The retrieval result with traditional measurement method and multiagent method is compared under the three weather conditions.

4.1. Retrieval and Analysis of Atmospheric Refractivity under Sunny Weather Condition. According to the result of retrieval atmospheric refractivity (Figure 4), the value of atmospheric refractivity acquired by multiagent method is entirely well fitted with the value of atmospheric refractivity acquired by observation. The average observation error of 96 samples which were observed in 24 hours is 42 percent of $N$. And the 


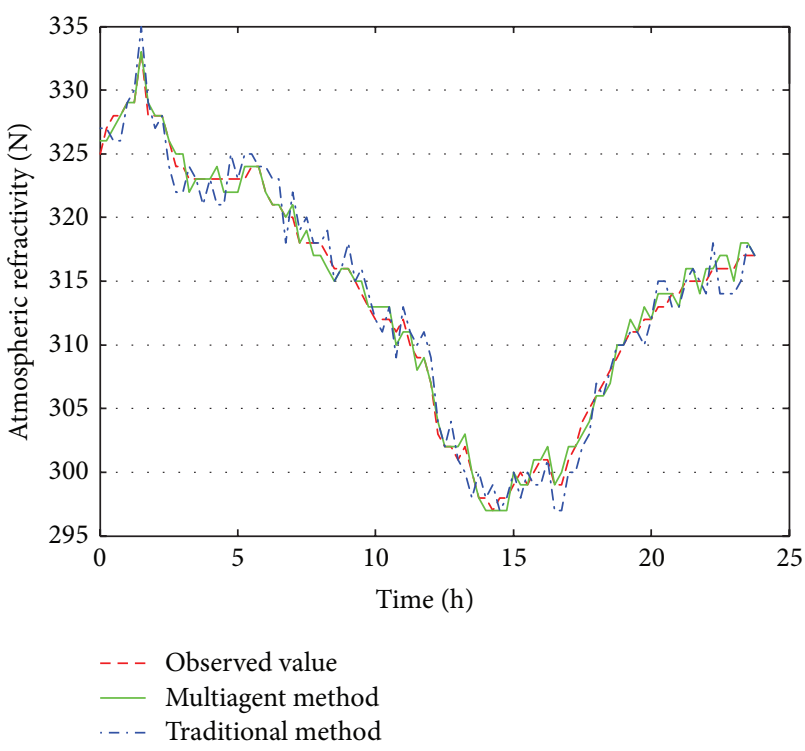

FIGURE 4: The comparison of the value of atmospheric refractivity $N$ under sunny weather condition. Red line: the value of atmospheric refractivity acquired by observation. Green line: the value of atmospheric refractivity acquired by multiagent method. Blue line: the value of atmospheric refractivity acquired by traditional method.

average observation error of all samples is less than 100 percent of $N$. The correlation coefficient of the observed samples and calculated samples is 0.993 , and the RMSE equals 1.072 .

The difference between retrieval atmospheric refractivity by multiagent method and traditional method changes slightly. It reflects the high sensitivity of the electromagnetic signals. It is meaningful to analyse mesoscale convective weather or fog of boundary changes and transport of water vapor.

4.2. Retrieval and Analysis of Atmospheric Refractivity under Foggy Weather Condition. According to the result of retrieval atmospheric refractivity (Figure 5), the value of atmospheric refractivity acquired by multiagent method is entirely well fitted with the value of atmospheric refractivity acquired by observation. The average observation error of 96 samples which were observed in 24 hours is 49 percent of $N$. And the average observation error of all samples is less than 100 percent of $N$. The correlation coefficient of the observed samples and calculated samples is 0.993 , and the RMSE equals 1.113 .

The range of observation error under foggy weather condition is very close to observation error under sunny weather condition. It means that tiny liquid water droplets do not cause interference to retrieval results. And it is feasible to retrieve atmospheric refractivity by measuring the electromagnetic signals using multiagent system. Meanwhile according to retrieval, it can be found that the atmospheric refractivity would increase rapidly prior to significantly reducing visibility to develop into a very dense fog. Observation of this phenomenon can be used as early warning of approaching fog important reference index.

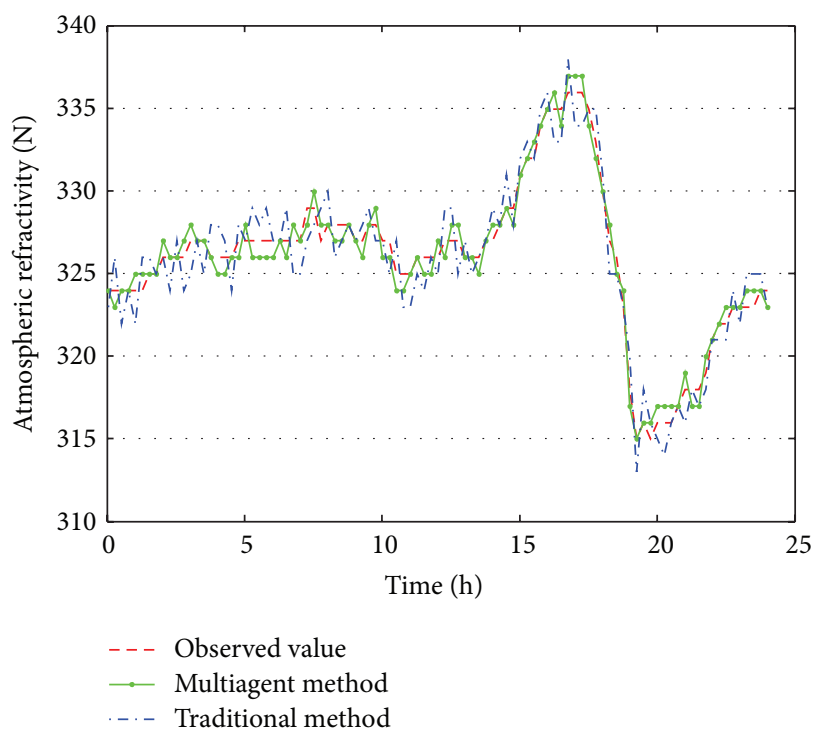

FIGURE 5: The comparison of the value of atmospheric refractivity $N$ under foggy weather condition. Red line: the value of atmospheric refractivity acquired by observation. Green line: the value of atmospheric refractivity acquired by multiagent method. Blue line: the value of atmospheric refractivity acquired by traditional method.

4.3. Retrieval and Analysis of Atmospheric Refractivity under Rainy Weather Condition. According to the result of retrieval atmospheric refractivity (Figure 6), the value of atmospheric refractivity acquired by multiagent method is entirely well fitted with the value of atmospheric refractivity acquired by observation. The average observation error of 96 samples which were observed in 24 hours is 57 percent of $N$. And the average observation error of all samples is less than 100 percent of $N$. The correlation coefficient of the observed samples and calculated samples is 0.991 , and the RMSE equals 1.091 .

Compared with the previous analysis, larger liquid water droplets do not interfere with the results of retrieval under rainy weather condition. Trends in rainfall and atmospheric vapor pressure of the index of refraction are consistent trends. Subtle changes in atmospheric refractivity of water vapor, temperature, and barometric pressure are very sensitive. The experimental validation of the method of the refractivity of atmospheric retrieval weather conditions in rainfall is possible and obtains higher retrieval accuracy.

\section{Conclusions and Future Work}

The refractivity of the low-altitude atmospheric inversion results fits well the results of the calculation of meteorological elements under sunny, foggy, and rainy weather condition according to the comparison. Tiny water droplets in the atmosphere almost have no impact on the results of the electromagnetic signal inversion of atmospheric refractive index. Therefore, electromagnetic measurement method based on multiagent is able to achieve real-time monitoring of water vapor near the ground changes under different weather 


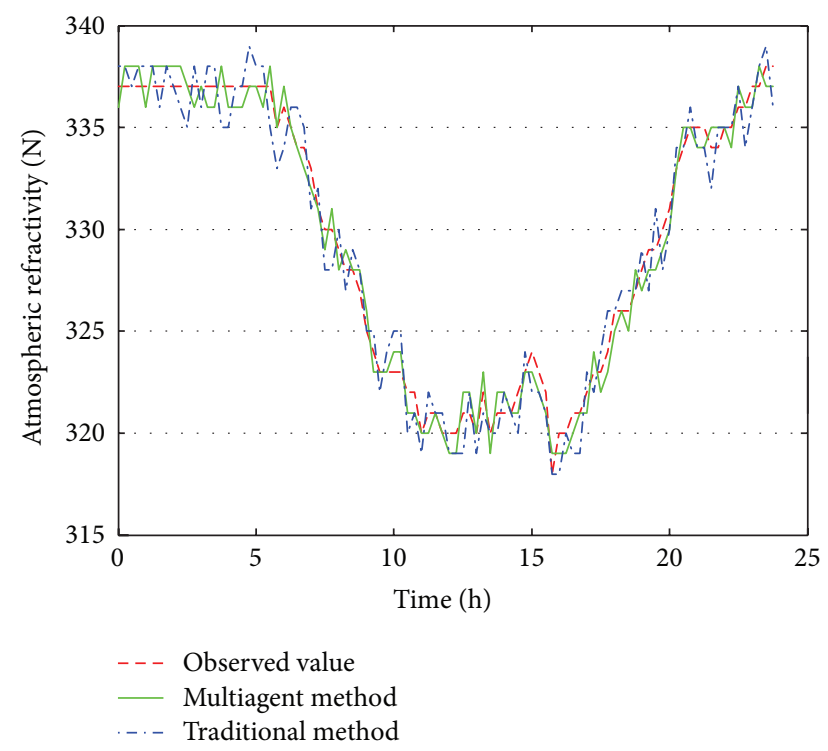

FIGURE 6: The comparison of the value of atmospheric refractivity $N$ under rainy weather condition. Red line: the value of atmospheric refractivity acquired by observation. Green line: the value of atmospheric refractivity acquired by multiagent method. Blue line: the value of atmospheric refractivity acquired by traditional method.

conditions with a high time resolution. It exhibits a good electromagnetic wave signal variation of the moisture sensitivity of the results obtained in comparison with the conventional method results consistent.

A certain accuracy of moisture content of the boundary layer method by multiagent can be achieved according to the experiment. In future work, the following points could be improved.

(1) Sensor agents are mounted on the car body in the experiment, so the trajectory of the sensor agents is restricted in the trajectory of the car. The data only generated in the position where car can reach. Although the interpolation method can be used to fill with the position where the car cannot reach, this is not the actual measured data. Sensor agents will be settled to aerocraft in further work. And track of aerocraft would be controlled by manager agents. The more comprehensive data will be acquired.

(2) The only specific frequency which is electromagnetic is used in experiment. Previous studies showed that electromagnetic wave of different frequency has different level of sensitivity to water vapor which depends on the wavelength. Shorter wavelength electromagnetic would be selected to compare with result in further work.

(3) It would be worthy to research location sampling algorithm of sensor agents which are controlled by manager agents.

\section{Conflict of Interests}

The authors declare that there is no conflict of interests regarding the publication of this paper.

\section{References}

[1] F. Fabry, "The spatial variability of moisture in the boundary layer and its effect on convection initiation: project-long characterization," Monthly Weather Review, vol. 134, no. 1, pp. 79-91, 2006.

[2] B.-G. Jeon, Y. Moon, and T.-W. Ahn, "A study on $11 \mathrm{MHz} \sim$ $1537 \mathrm{MHz}$ DCO using tri-state inverter for DAB application," in Proceedings of the IEEE Region 10 Conference (TENCON '09), pp. 1-5, Singapore, January 2009.

[3] J. Braun, C. Rocken, and R. Ware, "Validation of line-of-sight water vapor measurements with GPS," Radio Science, vol. 36, no. 3, pp. 459-472, 2001.

[4] B. L. Cheong, R. D. Palmer, T. Y. Yu, and C. Curtis, "Refractivity measurements from ground clutter using the National Weather Radar Testbed phased array radar," in Proceedings of the 32nd Conference on Radar Meteorology, Albuquerque, NM, USA, 2005.

[5] I. Martí, V. R. Tomás, L. A. García, and J. J. Martínez, "A multiagent system for managing adverse weather situations on the road network," Integrated Computer-Aided Engineering, vol. 17, no. 2, pp. 145-155, 2010.

[6] C. G. Someda, Electromagnetic Waves, CRC Press, New York, NY, USA, 2nd edition, 2006.

[7] J. Duan, M. Bevis, P. Fang et al., "GPS meteorology: direct estimation of the absolute value of precipitable water," Journal of Applied Meteorology, vol. 35, no. 6, pp. 830-838, 1996.

[8] A. E. Niell, A. J. Coster, F. S. Solheim et al., "Comparison of measurements of atmospheric wet delay by radiosonde, water vapor radiometer, GPS, and VLBI," Journal of Atmospheric and Oceanic Technology, vol. 18, no. 6, pp. 830-850, 2001.

[9] P. Miidla, K. Rannat, and P. Uba, "A mathematical model of troposphere water vapor tomography," in Proceedings of the 2nd International Conference on Environmental and Computer Science (ICECS '09), pp. 183-187, Dubai, UAE, December 2009.

[10] S. Padmanabhan, S. C. Reising, J. Vivekanandan, and F. Iturbide-Sanchez, "Retrieval of atmospheric water vapor density with fine spatial resolution using three-dimensional tomographic inversion of microwave brightness temperatures measured by a network of scanning compact radiometers," IEEE Transactions on Geoscience and Remote Sensing, vol. 47, no. 11, pp. 3708-3721, 2009.

[11] R. J. Watson and C. J. Coleman, "The use of signals of opportunity for the measurement of atmospheric refractivity," in Proceedings of the 4th European Conference on Antennas and Propagation (EuCAP '10), pp. 1-5, April 2010.

[12] W. Xu, R. Lv, X. Yu, Z. Yang, and L. Deng, "Synchronous orthogonal DAB spectrum layout scheme," in Proceedings of the 2nd International Conference on Biomedical Engineering and Informatics (BMEI'09), October 2009.

[13] V. R. Tom and L. A. Garc, "A cooperative multiagent system for traffic management and control," in Proceedings of the 4th International Joint Conference on Autonomous Agents and Multi-Agent Systems (AAMAS '05), pp. 52-59, Utrecht, The Netherlands, 2005.

[14] Z. Yan, D. Chakraborty, A. Misra, H. Jeung, and K. Aberer, "Sammple: detecting semantic indoor activities in practical 
settings using locomotive signatures," in Proceedings of the International Symposium on Wearable Computers, pp. 37-40, Newcastle, UK, 2012.

[15] J. Zhu, P. Wu, X. Wang, and J. Zhang, "SenSec: mobile security through passive sensing," in Proceedings of the International Conference on Computing, Networking and Communications (ICNC '13), pp. 1128-1133, IEEE, San Diego, Calif, USA, January 2013.

[16] D. Hasenfratz, O. Saukh, S. Sturzenegger, and L. Thiele, "Participatory air pollution monitoring using smartphones," in Proceedings of the 1st International Workshop on Mobile Sensing: From Smartphones and Wearables to Big Data, 2012.

[17] M. Faulkner, M. Olson, R. Chandy, J. Krause, K. M. Chandy, and A. Krause, "The next big one: detecting earthquakes and other rare events from community-based sensors," in Proceedings of the 10th International Conference on Information Processing in Sensor Networks (IPSN '11), pp. 13-24, Chicago, Ill, USA, April 2011.

[18] P. Wu, H.-K. Peng, J. Zhu, and Y. Zhang, "Senscare: semiautomatic activity summarization system for elderly care," in International Conference on Mobile Computing, Applications, and Services (MobiCASE '11), 2011.

[19] M. Rinard and M. Lam, "The design, implementation, and evaluation of Jade," ACM Transactions on Programming Languages and Systems, vol. 20, no. 3, pp. 483-545, 1998.

[20] M. Proctor, "Relational declarative programming with JBoss Drools," in Proceedings of the 9th International Symposium on Symbolic and Numeric lgorithms for Scientific Computing (SYNASC '07), Timisoara, Romania, September 2007. 


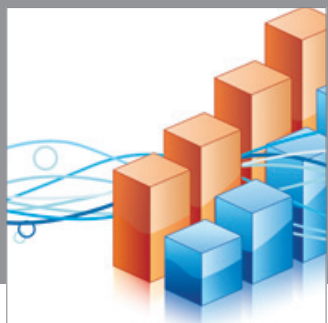

Advances in

Operations Research

mansans

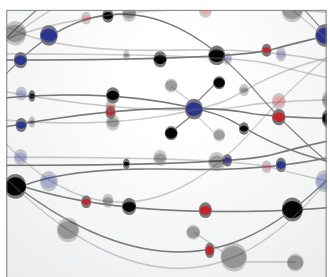

The Scientific World Journal
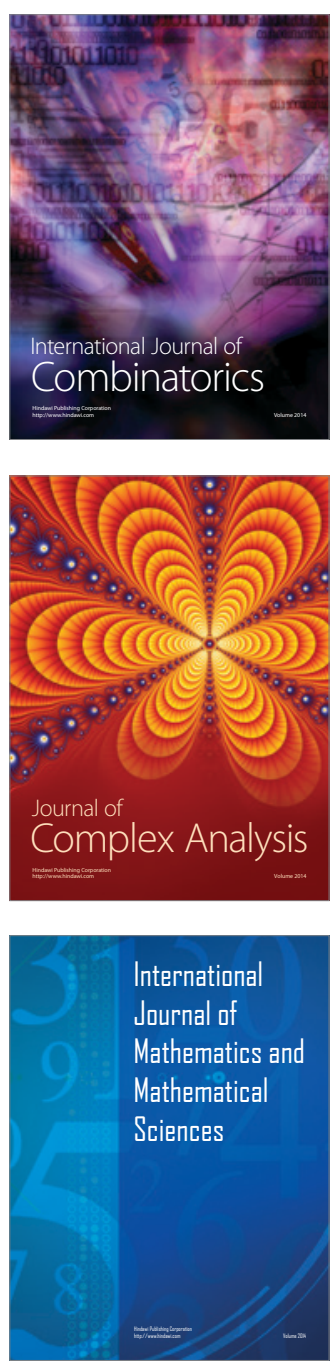
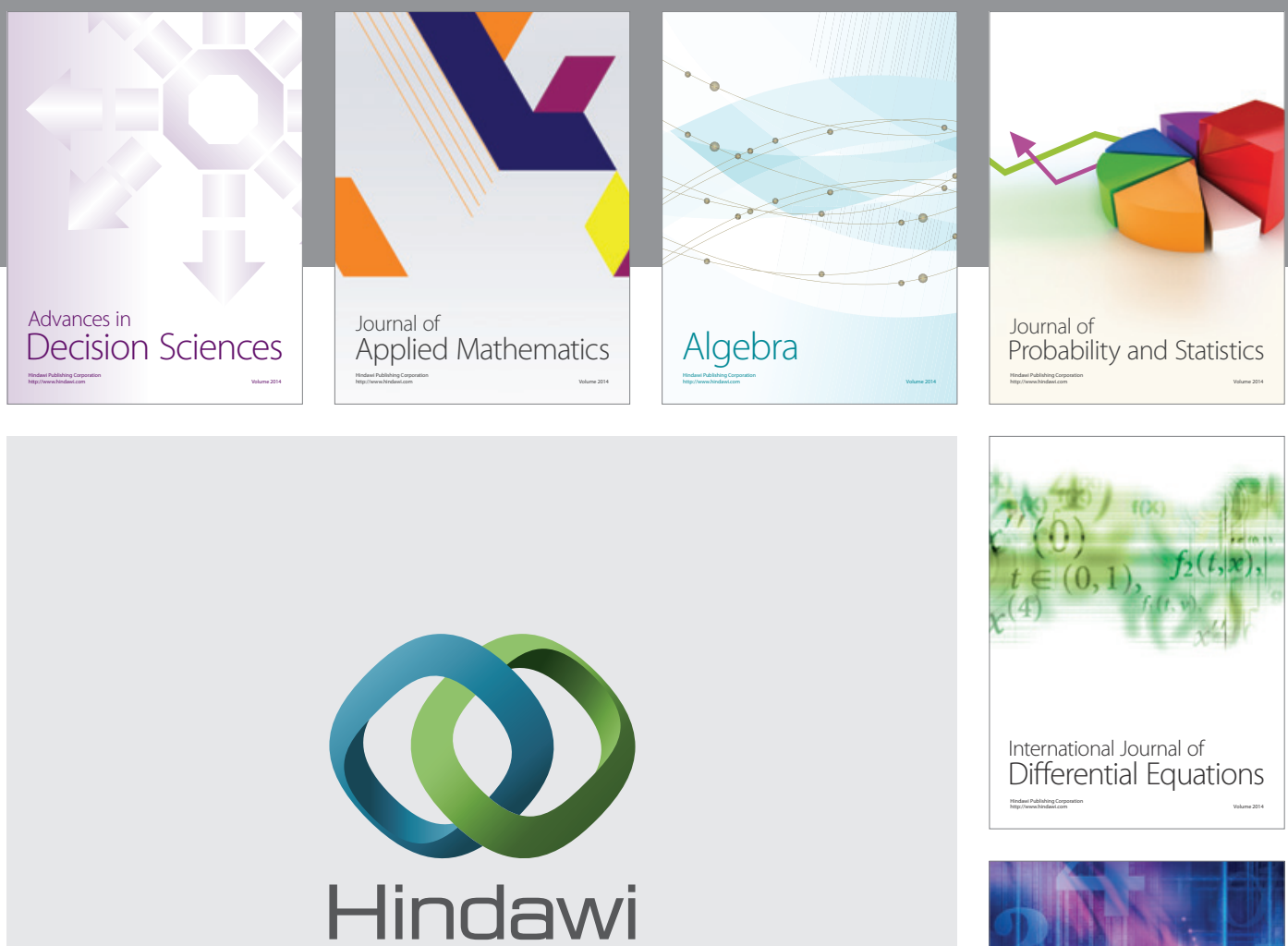

Submit your manuscripts at http://www.hindawi.com
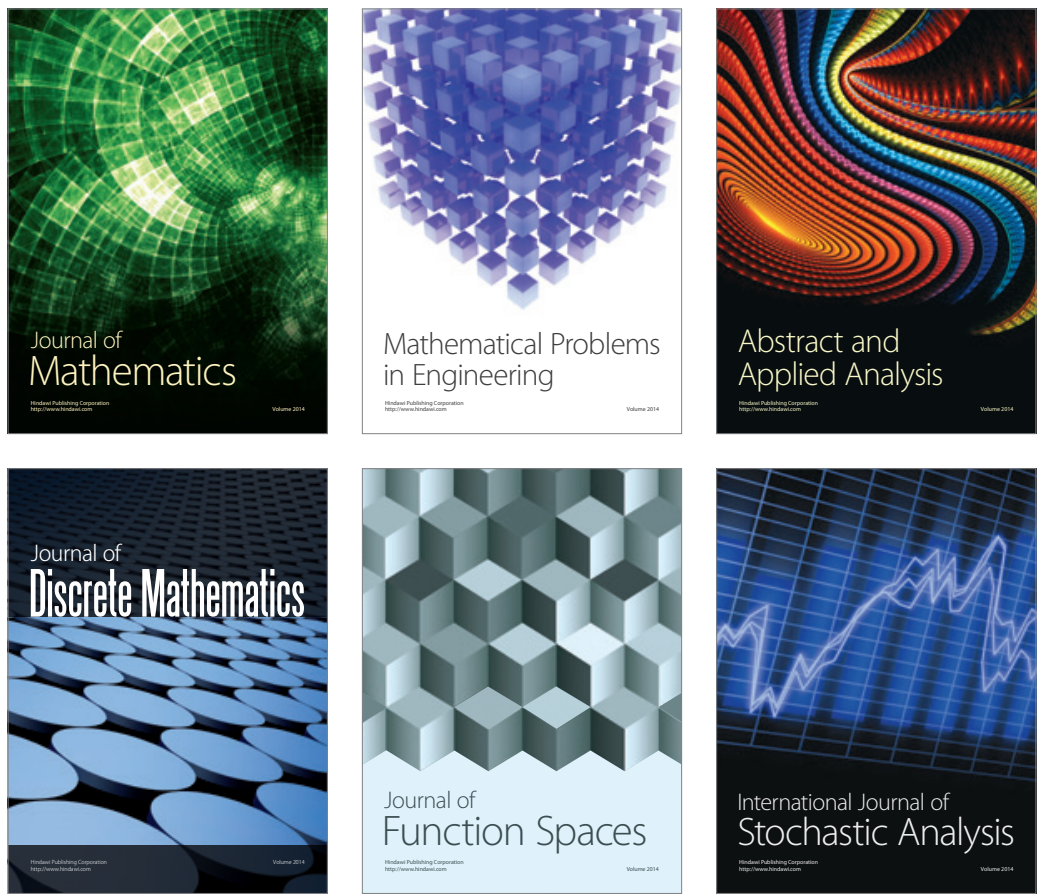

Journal of

Function Spaces

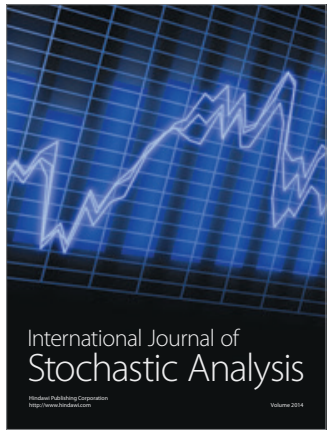

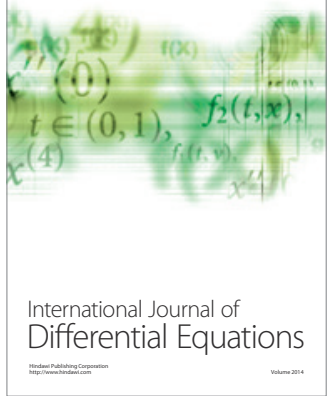
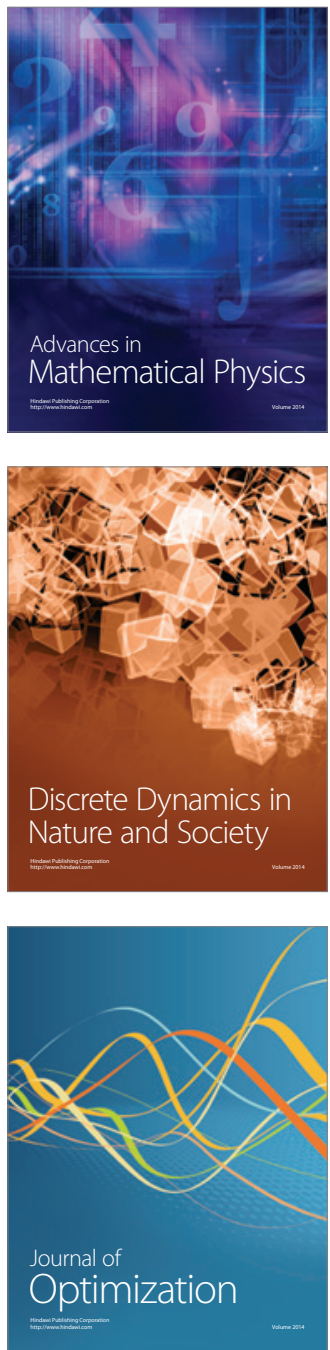